6 Science and Engineering Research Council

Rutherford Appleton Laboratory

Chilton DIDCOT Oxon OX11 0QX

RAL-90-056

s)

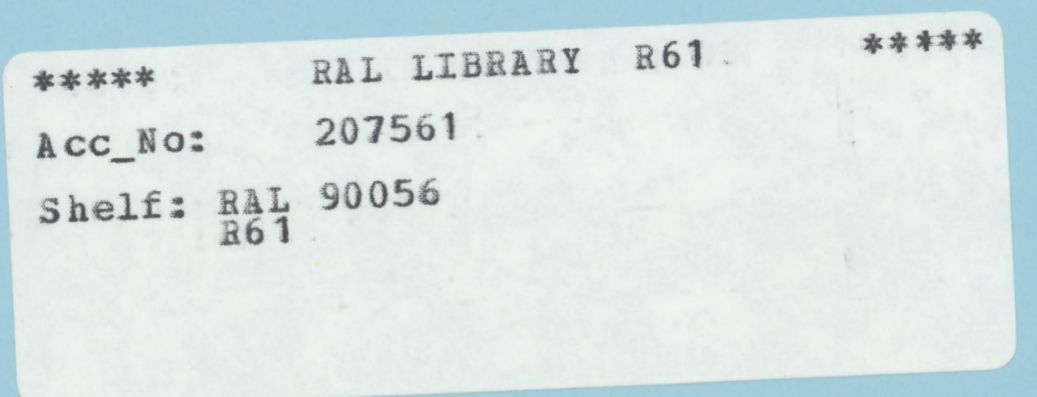

Theory of Wave Activity Occuring in the AMPTE Artificial Comet

R Bingham V D Shapiro V N Tsytovich U de Angelis $M$ Gilman and V S Shevchenko

August 1990

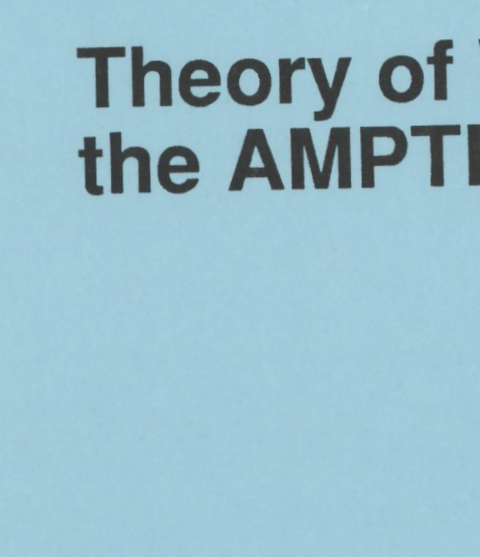

LIBRARY

RUTHERFORD

11 ASEP 1990

\&ABORATORY 
(C) Science and Engineering

Research Council 1990

'The Science and Engineering Research Councll does not accept any responsibility for loss or damage arising from the use of information contained in any of its reports or in any communication about its tests or investigations.' 


\title{
Theory of Wave Activity Occurring in the AMPTE Artificial Comet
}

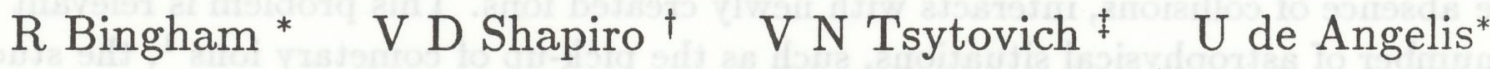 \\ M Gilman ${ }^{\dagger}$ and V S Shevchenko ${ }^{\dagger}$
}

\begin{abstract}
One of the main experiments of the Active Magnetospheric Particle Tracer Explorers (AMPTE) satellite mission was the release of neutral barium atoms in the solar wind. The barium atoms ionized by photoionisation extremely rapidly forming a dense, expanding, plasma cloud which interrupted the solar wind flow creating diamagnetic cavities. On the upstream side of the cavity a region of compressed plasma and enhanced magnetic field was created as the result of being produced by the slowing down and deflection of the solar wind, and magnetic field line draping. Intense electrostatic and magnetic turbulence was observed by both the IRM and UKS satellites at the boundary of the diamagnetic cavity with the most intense being detected near the outer boundary of the compressed region. In this paper we examine how the newly created expanding plasma couples to the solar wind by means of plasma beam and current driven instabilities. In particular we show how lower-hybrid and lower-hybrid drift waves are generated by crossfield proton-barium streaming instabilities and cross field electron currents. The saturation mechanism for these waves is considered to be the modulational instability, this instability can also lead to filamentation and coupling to magnetosonic modes which are also observed. As the result of modulational instability the $k_{\|}$component increases, which allows the heating and acceleration of electrons that is consistent with the observations.
\end{abstract}

*Rutherford Appleton Laboratory, Chilton, Didcot, Oxon, OX11 0QX

${ }^{\dagger}$ Space Research Institute, USSR Academy of Sciences, Moscow

${ }^{\ddagger}$ General Physics Institute, USSR Academy of Sciences, Moscow 


\section{Introduction}

Recent experiments carried out by the Active Magnetospheric Particle Tracer Explorers (AMPTE) involved the release of lithium and barium atoms in the solar wind. In all cases the expanding cloud of atoms was photoionized by solar ultra-violet radiation, producing an obstacle to the solar wind flow and the formation of diamagnetic cavities. One of the main objectives of the experiments was to understand how the solar wind, in the absence of collisions, interacts with newly created ions. This problem is relevant to a number of astrophysical situations, such as the pick-up of cometary ions ${ }^{1}$, the study of planetary ion exospheres interacting with the solar wind ${ }^{2}$ and the pick-up of helium and oxygen ions of interstellar or planetary origin ${ }^{3}$. Since both the released plasma and solar wind plasma are collisionless any interaction between them must depend on wave-particle interactions producing the momentum coupling.

The Ampte releases provide at the present time the best detailed data set for the study of artificially created plasma comets and in general for the investigation of plasma turbulence created by releases and in flows in the solar wind. Using the results makes it possible to try to formulate a physically self-consistent picture of the whole event including especially the different turbulent modes excited and the creation of accelerated electrons. At the present time some of the important parts of this picture have been considered such as the possible excitation mechanisms for lower-hybrid waves ${ }^{4,5}$ and the subsequent electron acceleration by them ${ }^{4,6}$. However, many aspects of the interaction are still waiting to be understood even qualitatively, such as the observed level of ionsound turbulence inside the cavity ${ }^{7}$, the current filamentary structure, the slowing down and heating of the solar wind protons and the heating of the barium ions ${ }^{8}$. We believe that for different parts of the observed phenomena several explanations can be given. We would like, though, to construct a general picture which will be able to give qualitative answers to the most important observations, if not all, and find out the relationship between the different phenomena observed such as the nature and level of fluctuations observed both inside the diamagnetic cavity, at the boundary between the cavity and the shock-like structure and within the shock-like structure. It seems very likely that all these processes are related making it less likely that a number of different processes are responsible. In the present paper we propose one main self-consistent picture of the turbulence excited by the Ampte release. Such a model will help in the understanding of future possible experiments. A similar approach can be used for the bow-shock, aurora and other precipitation phenomena. In considering a self-consistent approach we bear in mind the necessity of estimating the most probable nonlinear processes going on in a consistent manner. 
One important point should be mentioned before we start the discussion, namely that one could not expect from the point of view of the present understanding of nonlinear turbulent process that the broad spectra observed are strongly related to the frequencies of linear modes or to the wavelengths of the fastest growing modes one can only obtain best estimates for such values. The opposite should be expected, namely due to nonlinear processes the turbulence should have a broad spectra with a maximum intensity not at the wavelengths of the maximum growthrates, or at the frequency corresponding to the maximum growthrate, most of the observed features will be the result of turbulent nonlinear processes with a few features being describable by linear theory.

\section{Major observational data on the Ampte barium release}

Initially before the barium release the solar wind parameters were electron density $n_{e} \simeq 2 \mathrm{~cm}^{-3}$, velocity of solar wind $v_{s w} \simeq 550 \mathrm{~km} / \mathrm{sec}$, electron temperature and magnetic field $T_{e} \approx 20 e v, B \approx 10^{-4} G$ or $10 n T$. For these parameters the Debye length is $20 \mathrm{~m}$ and the lower-hybrid resonance frequency $\omega_{L H} \approx\left(\omega_{H i} \omega_{H e}\right)^{\frac{1}{2}} \simeq 20 \mathrm{sec}^{-1}$. The interaction of released ions with the solar wind produces a diamagnetic cavity, of the order of $70 \mathrm{~km}$ in radius, surrounded by a pile-up region of compressed plasma and high magnetic field strength of the order of $100 \mathrm{nT}^{9}$. The scale size of the magnetic field structure is of the order of $1-10 \mathrm{~km}$ or less, with a gradual increase on a scale length of up to $200 \mathrm{~km}$. The magnetic structure surrounding the cavity is influenced by the solar wind and is very filamentary ${ }^{9}$. The size of the filamentary structures could be less than $1 \mathrm{~km}$ or even $100 \mathrm{~m}$. The cavity is surrounded by a shock-like structure in the upstream region where there exists a large magnetic field structure of the order of $60-100 \mathrm{nT}$. The lower-hybrid frequency for barium ions in this region is of the order of $30-100 \mathrm{~Hz}$, which corresponds to the range of frequencies of intense electrostatic noise observed mainly in the region surrounding the cavity or between the cavity and the upstream solar wind. The plasma density of the barium ions (singly ionized barium, ie the density of cold electrons appearing in the cavity is of the order of the density of barium ions) varies from $10^{5}-10^{4} \mathrm{~cm}^{-3}$ in the centre down to almost the solar wind density at the upstream side of the cavity. Inside the cavity ion sound turbulence is observed and maintained for a period of observation (10 min.) although the Landau damping of ion sound waves is of the order of or less than 1/10 of a second, this demonstrates a necessity for a source driving the ion sound turbulence. The wave turbulence in the cavity is at a very low level, in comparison with the compressed region as shown in figure 3 of reference 5 . The level of the turbulent fields observed will be given below in connection with theoretical estimates.

In the region of the large field compression at the front of the cloud and more importantly in the whole of the turbulent region between the shock and the cavity 
electron energization (acceleration) is observed. Electrons with energies greater than $100 \mathrm{eV}$ are created. Solar wind ions are slowed down and deflected in this region. The most intense wave activity is also observed in the region of enhanced compression. The slowing down of the solar wind to $270 \mathrm{~km} / \mathrm{sec}$ also occurs in this region corresponding to a flow energy of $0.4 \mathrm{keV}$. The initial flow energy is about $1.6 \mathrm{keV}$. This means that the solar wind loses about $70 \%$ of its flow energy and it is heated up to $0.4 \mathrm{keV}$ at the rear of the wave activity. The barium ions can be considered practically as not moving. The most intense waves appear at a frequency of $30 \mathrm{~Hz}$ at 14:30 the time of maximum magnetic field activity encountered.

\section{A general physical picture of the wave activity}

It is obvious that in the strongly turbulent region the magnetic fields are important and the interaction of solar wind protons with barium ions should be treated with the magnetic field taken into account particularly since some of the wave activity observed is in the range of lower-hybrid frequencies ie some of the modes excited require the presence of the magnetic field (in this sense the analysis of reference ${ }^{7}$ is not quite adequate to describe the whole problem). Instead of a reflected ion beam as at the Earth's bow shock, the two beam situation in the barium release appears due to the interaction of the solar wind protons with the almost stationary barium ions.

This ion-ion beam interaction should excite two types of waves, a long wavelength mode $k \simeq \omega_{p e} / c$ in the vicinity of the lower-hybrid frequency and the shorter wavelength $k \sim \omega_{p e} / v_{T e}$ ion acoustic mode. The most intensive wave activity is to be connected with the ion-acoustic mode, the frequency of this mode as measured by the spacecraft varies over a wide range from tens of $\mathrm{Hz}$ up to $\mathrm{kHz}$. Simple estimates based on quasilinear theory give for the wave electric field spectral density the value $10-30 \mathrm{mV} / \mathrm{m} \sqrt{\mathrm{Hz}}$, the growth rate increases with $n_{B}$ - the density of the barium beam and as a consequence the instability mechanism under consideration is important in the whole turbulent zone between the shock and the cavity. The effective excitation of the lower-hybrid mode is possible only in the case of oblique propagation with respect to the magnetic field. The excitation takes place in the outer part of the turbulent zone where $n_{B} \simeq n_{o} \frac{m_{B}}{m_{p}}, m_{B, P}$ is barium; proton mass. For larger $n_{B}$ the growth rate of the lower-hybrid mode $\gamma$ decreases as $n_{B}^{-\frac{1}{3}}$ thus making the excitation of this mode at $n_{B} \sim 10^{4}-10^{5}$ impossible since the time needed for excitation $10 / \gamma$ becomes larger than the convective time $L / u(L \simeq 300 \mathrm{~km}$ - the width of the turbulent zone, $u \simeq 270 \mathrm{~km} / \mathrm{s}$ the speed of the solar wind slowed down by the cavity).

Another type of instability to be considered is the lower-hybrid drift instability ${ }^{10}$ induced by the currents. The largest currents are the diamagnetic currents $\left(\mathbf{J}_{D}=\right.$ 
$\left.-\underline{\nabla}(n T) \times \mathrm{B} / B^{2}\right)$ existing at the boundary of the cavity. The growth rate of this instability is limited only by the timescale during which the current flows and has therefore less rigid conditions of applicability than the proton-barium beam instability, ie it can also occupy the whole turbulent region. It also exists in the region of high ion (barium) density and is not suppressed with increasing density. But there does exist a threshold for the drift velocity of the current carrying electrons to drive this instability. This threshold is not large - the electron drift velocity necessary could be much less than the ion thermal velocity. The saturated level of electrostatic fluctuations excited by both types of lower-hybrid instabilities are, as estimations show, determined by the modulational instability, which is somewhat different from the usual modulational instability because of the kinetic pressure of the barium ion flow in the solar wind frame. In this case the nonlinear coupling between the lower hybrid waves occurs through the low frequency magnetic field perturbations. Slowly varying magnetic field structures having wavenumbers $k \leq \omega_{p e} / c$ which corresponds to characteristic space scales of about $1 \mathrm{~km}$ or less should develop. In places of low magnetic field the frequency of the lowerhybrid mode is decreasing, hence these places serve as potential wells in which the wave quanta of the lower-hybrid mode can be trapped. This trapping results in the modulation of the lower-hybrid wave intensity which further increases modulation of the magnetic field under the action of the pondermotive force of the wave.

The presence of fine scale low frequency magnetic field structures follows from observations obtained by Lühr et $\mathrm{al}^{9}$. The creation of these magnetic field structures due to the modulational instability of lower-hybrid waves will result, according to Ampere's Law, in currents with $\underline{J}=\frac{c}{4 \pi} \underline{\nabla} \times \underline{B}$ sufficient to excite the lower-hybrid drift modes. It is important that the development of the modulational instability lead to the appearance of waves with $k_{\|} \simeq\left(\frac{m_{e}}{m_{B}}\right)^{\frac{1}{2}} k_{L H}$, which can be absorbed by the tail of the electron distribution. In the initial stage of the instability one could divide the turbulent region between the solar wind and the inner boundary of the cavity into two regions. In the outer part the instability of lower-hybrid waves driven by the two beam instability (solar wind proton plus the barium ions) develops. In the inner region the lower-hybrid drift waves are excited by the diamagnetic currents flowing as a consequence of Ampere's law due to the fine scale magnetic field structures.

Finally the stage will be reached when in the whole turbulent region including that part where initially only the beam driven lower-hybrid instability develops the lowerhybrid drift mode will also exist. These lower-hybrid waves of both types are absorbed by electrons due to Landau damping and energize them. The time of energization should be less than the confinement time of electrons in the turbulent region. In this context it is important that lower-hybrid drift modes exist in the whole turbulent region. 
Otherwise for the usual lower-hybrid instability, the outer part of the turbulent region where it is effectively excited seems to be insufficient in size to accelerate the electrons. The fastest electrons are lost thus increasing the electrostatic potential of the turbulent region up to their escape energy (say 100ev). This D.C. electric field cannot produce a large current due to the large value of the effective collision frequency inside the turbulent region. These processes can also explain the heating of protons observed, first of all via the beam-plasma driven ion-acoustic instability. Since the ion acoustic instability considered in this paper is of the negative energy type, in the process of increasing of their amplitudes the waves heat the resonant particles (barium or proton ions) by an amount equal to their energy gain.

The proposed mechanisms are therefore the development of broad band intense electrostatic turbulence as the result of the ion-acoustic instability as well as lower-hybrid turbulence that could also be responsible for fine scale magnetic field structures. Both instabilities are driven by barium beam solar wind plasma interaction. The currents associated with the magnetic field structures could drive another type of lower-hybrid instability - namely lower-hybrid drift instability. This turbulence is finally absorbed by fast electrons, explaining the appearance of high energetic electron tails in the turbulence zone.

The above scenario will be similar to the case of a shock and particle precipitation. Indeed in the case of a bow shock the role of the second beam is played by the protons reflected by the shock and in the case of precipitation the flux of fast ions in the beam. The lower-hybrid drift waves appear at the final stage and can also explain the filamentary structure of magnetic fluctuations at the shock and perhaps also the filamentary structure of aurora.

\section{Linear theory of the various instabilities}

A) Proton-barium beam plasma instability.

We start from the investigation of long wavelength instabilities $\left(k \sim \omega_{p e} / c\right)$ resulting in the excitation of waves with the frequencies in the vicinity of the lower-hybrid resonance. Electrons are magnetized in such oscillations where $k v_{T e} \ll \omega_{H e}$, and $\omega \ll \omega_{H e}$. The main plasma is the ionized barium gas, solar wind protons form a cold beam in such a plasma. The dispersion relation for oblique $\left(k_{\|} \neq 0\right)$ waves excited due to beam - plasma interaction has the form:

$$
\frac{\omega_{L H}^{2}}{\omega^{2}} \frac{m_{p}}{m_{B}}+\frac{\omega_{H e}^{2}}{\omega^{2}} \frac{k_{\|}^{2}}{k^{2}+\omega_{p e}^{2} / c^{2}}+\frac{\omega_{L H}^{2}}{\left(\omega-\mathbf{k} \cdot \mathbf{v}_{s w}\right)^{2}} \frac{n_{p}}{n_{B}}=1+\frac{\omega_{p e}^{2}}{k^{2} c^{2}}
$$

Here $\omega_{L H}=\sqrt{\omega_{H e} \omega_{H p}}$ is the lower-hybrid frequency in a dense $\left(\omega_{p e} \gg \omega_{H e}\right)$ solar wind plasma, $n_{B}, n_{p}, m_{B}$, and $m_{p}$ are the densities and masses for barium ions and protons 
respectively. For the two species case (protons flowing through electrons) this equation was obtained and analysed in detail in ref $^{12}$ and for the plasma and magnetic field inhomogeneities taken into account in ref $^{13}$. The effect of inhomogeneity leading to the lower-hybrid drift instability will be analysed in the next section. Here we will restrict ourselves by analysing the homogeneous case and our aim would be to understand how the presence of a third (barium) component will influence the modified two stream instability described by equ. 1 . We consider the region where $n_{B} \gg n_{p}$ and hence use the condition of quasi neutrality in the form $n_{e} \simeq n_{B}$ ie the electron density is balanced by the barium ion density. The plot of the left hand side of the Eq.1 F $(\omega, k)$ as a function of $\omega$ is shown in Figure 1. The minimal value of $F$ for the system to be stable is given by

$$
F_{\min }=\frac{n_{p}}{n_{B}} \frac{\omega_{L H}^{2}}{\left(\mathbf{k} \cdot \mathbf{v}_{s w}\right)^{2}}\left(1+\alpha^{\frac{1}{3}}\right)^{3}
$$

where the following notation

$$
\alpha\left(k, k_{\|}\right)=\frac{n_{B}}{n_{p}} \frac{m_{p}}{m_{B}}+\frac{k_{\|}^{2}}{\left(k^{2}+\omega_{p e}^{2} / c^{2}\right)} \frac{m_{p}}{m_{e}} \frac{n_{B}}{n_{p}}
$$

is used.

For the instability the dispersion relation equation (1) must have at least two complex roots the condition for that is when $\mathrm{F}$ is less than $F_{M I N}$ which corresponds to

$$
\text { k. } \mathbf{v}_{s w}<k v_{A}\left(\frac{n_{p}}{n_{B}}\right)\left(1+\alpha^{\frac{1}{3}}\left(k, k_{\|}\right)\right)^{\frac{3}{2}}
$$

Here $v_{A}=H_{o} / \sqrt{4 \pi n_{p} m_{p}}$, is the Alfven velocity in the solar wind plasma. For transverse magnetic field propagation $\left(k_{\|}=0\right), \alpha \ll 1$ corresponding to the situation in the AMPTE barium release. The condition for waves propagating almost along the beam direction $\left(\mathbf{k}_{\|} \mathbf{v}_{s w}\right)$ has the form

$$
n_{B}<n_{p} \frac{v_{A p}}{v_{s w}}
$$

In the case $n_{p}=n_{e}$ it coincides with the condition obtained in ref. ${ }^{12}$. It follows from this relation that for the conditions of the AMPTE experiment the instability under consideration is possible only in the outer part of the barium cloud where

$$
n_{B}<(5-10) n_{p}
$$


The wavenumber of the fastest growing mode in the case $\alpha \ll 1$ is equal to:

$$
k=\sqrt{\frac{\omega_{L H}^{2}}{v_{s w}^{2}} \frac{n_{p}}{n_{B}}-\frac{\omega_{L H}^{2}}{v_{A p}^{2}} \frac{n_{B}}{n_{p}}}
$$

The frequency and the growth rate of that mode are obtained from the relation

$$
\omega=\left(\frac{\alpha}{2}\right)^{\frac{1}{3}} \frac{1+i \sqrt{3}}{2} \mathbf{k} \cdot \mathbf{v}_{s w}
$$

In a more dense part of the cloud $n_{B} \leq n_{p} \frac{m_{B}}{m_{p}} \simeq 10^{2} n_{p}$ it follows form Eq. (3) that the instability is possible only for the case where $\alpha \gg 1$ or for oblique $\left(k_{\|} \neq 0\right)$ waves. For the case $n_{e}=n_{p}$ the instability of oblique waves was analysed in ref. ${ }^{13}$. The frequencies of excited waves for the case $\alpha \gg 1$ are obtained from the relation

$$
\omega=\frac{\omega_{L H}}{\sqrt{1+\omega_{p e}^{2} / k^{2} c^{2}}} \sqrt{\frac{m_{p}}{m_{B}}+\frac{k_{\|}^{2}}{k^{2}+\omega_{p e}^{2} / c^{2}} \frac{m_{p}}{m_{e}}}
$$

For the waves with $k_{\|} / k \gg \sqrt{\frac{m_{e}}{m_{B}}}$, corresponding to oblique electrostatic waves with $\omega=\omega_{H e} k_{\|} / k$ for $\omega_{p e} \ll k c$, and the whistler mode with $\omega=\omega_{H e} \frac{c^{2} k_{\|} k}{\omega_{p e}^{2}}$ for $\omega_{p e} \gg k c$. The growth rate of the most unstable mode for $\alpha \gg 1$ is also easily obtained from the Eq.(1) and is given by

$$
\gamma=\frac{\sqrt{3}}{2^{\frac{4}{3}}} \frac{\omega}{\alpha^{\frac{1}{3}}\left(k, k_{\|}\right)}
$$

For example for the AMPTE conditions where $v_{A} \simeq 2 \times 10^{8} \mathrm{~cm} / \sec \left(H_{o} \simeq 100 \mathrm{nT}\right), v_{s w} \sim$ $2.7 \times 10^{7} \mathrm{~cm} / \mathrm{s}$ the excitation of waves in the region $n_{B}=n_{p} m_{B} / m_{p}$ is possible in the case $\alpha \geq 10^{2}\left(k_{\|} / k \geq \sqrt{\frac{m_{e}}{m_{p}}}\right)$, the frequency in this case is $\omega \geq \omega_{L H} \simeq 2.5 \times 10^{2} s^{-1}$. For $\omega \simeq \omega_{L H}, \alpha \simeq 10^{2}$ the growth rate obtained from Eq. (8) is $\gamma \sim 40 s^{-1}$ and the instability growth length $L \sim 10 v_{s w} / \gamma \sim 10^{7} \mathrm{~cm}$ is quite sufficient for the excitation of waves in the turbulent zone. In the more dense region further decrease of $\gamma(\gamma$ drops as $n_{B}^{-\frac{1}{3}}$ for fixed $\omega$ ) makes it impossible to excite the waves in that frequency range. The similar decrease of $\gamma$ makes the excitation of waves propagating at large angles $\theta$ towards the beam impossible. In this case it follows from Eq. (8) that the instability region is shifted towards larger $n_{B}$ but the growth rate decreases proportionally to $\cos \theta$ in accordance with Eq. (6). 
It is more convenient to consider the excitation of short wavelength ion-acoustic modes in the solar wind reference frame, in which the barium ions form a cold beam. Since the conditions $k v_{T e} \gg \omega_{H e}$ and $k_{\|} v_{T e} \gg \omega$ are fulfilled for that mode the electrons are unmagnetised. First we will consider the case of cold protons and $\omega \gg k v_{T p}$ then the dispersion relation has the following form

$$
1+\frac{1}{k^{2} d_{e}^{2}}-\frac{\omega_{p p}^{2}}{\omega^{2}}-\frac{n_{B}}{n_{p}} \frac{m_{p}}{m_{B}} \frac{\omega_{p p}^{2}}{\left(\omega+\mathbf{k} \cdot \mathbf{v}_{s w}\right)^{2}}=0
$$

where $d_{e}=\sqrt{\frac{T e}{4 \pi e^{2} n_{e}}}-$ is the electron Debye length, and $\omega_{p p}$ is the proton Langmuir frequency. First of all we shall consider the case $\alpha_{o} \equiv \frac{n_{B}}{n_{p}} \frac{m_{p}}{m_{B}} \ll 1$. In this case the frequency of the excited wave is the usual frequency of the ion-acoustic mode namely

$$
\omega_{s}=\frac{\omega_{p p} k d_{e}}{\sqrt{1+k^{2} d_{e}^{2}}} .
$$

The Cherenkov resonance condition with a beam becomes $\omega_{0} \simeq-\underline{k} \cdot \underline{v}_{s w}$ and it is easy to see that the excited waves propagate almost perpendicular to the ion beam with $\frac{\underline{\mathbf{k}} \cdot \mathbf{v}_{s w}}{\left|k v_{s w}\right|} \leq \frac{1}{v_{s w}} \sqrt{\frac{T_{e}}{m_{p}} \frac{n_{p}}{n_{B}}} \approx 10^{-1}-10^{-2}$. Substituting into the dispersion relation $\omega=-\underline{\mathbf{k}} \cdot \underline{\mathbf{v}}_{s w}+\delta \omega$, and using $|\delta \omega| \ll \omega_{o}$ we have from Eq.(9):

$$
\delta \omega=\omega_{o}\left(\frac{\alpha_{o}}{2}\right)^{\frac{1}{3}} \frac{1+i \sqrt{3}}{2}
$$

The condition of applicability of Eq. (9) is $\omega \gg k v_{T p}$ which with the help of Eq. (10) can be written in a form $\frac{T_{p}}{T_{e}} \ll \frac{n_{p}}{n_{B}}$ and it can only be fulfilled in the outer part of the cloud where the density of barium ions does not exceed very significantly the solar wind density. More importantly is the opposite case with $\omega \ll k v_{T p}$ when the beam driven instability is of a dissipative nature. In that case the dominant dissipation is due to the solar wind protons and the dispersion relation has the form

$$
\begin{gathered}
1+1 / k^{2} d_{e}^{2}+1 / k^{2} d_{p}^{2}+i \sqrt{\frac{\pi}{8}} \frac{\omega}{k v_{T p}} \frac{1}{k^{2} d_{p}^{2}} \\
-\alpha_{o} \frac{\omega_{p p}^{2}}{\left(\omega+\mathbf{k} \cdot \mathbf{v}_{s w}\right)^{2}}=0
\end{gathered}
$$

the solution of which is the following 


$$
\omega+\mathbf{k} \cdot \mathbf{v}_{s w}=-\frac{\alpha_{o}^{\frac{1}{2}} \omega_{p p} k d_{*}}{\sqrt{1+k^{2} d_{*}^{2}}}\left(1-i \sqrt{\frac{\pi}{8}} \frac{\omega}{k v_{T p}} \frac{d_{*}^{2} / d_{p}^{2}}{1+k^{2} d_{*}^{2}}\right)
$$

where we have used the notation

$$
\frac{1}{d_{*}^{2}}=\frac{1}{d_{p}^{2}}+\frac{1}{d_{e}^{2}}=4 \pi e^{2}\left(\frac{n_{p}}{T_{p}}+\frac{n_{e}}{T_{e}}\right)
$$

The condition for the instability developing

$$
\left|\mathbf{k} . \mathbf{v}_{s w}\right|>\frac{\alpha_{o}^{\frac{1}{2}} \omega_{p p} k d_{*}}{\sqrt{1+k^{2} d_{*}^{2}}}
$$

corresponds to a negative energy wave as is usual in the case of dissipative type instabilities. It follows from Eq. (13) that the growth rate of the instability increases with an increase in the barium ion density $\left(\gamma \sim n_{B}^{\frac{1}{2}}\right)$. The solution of the dispersion relation corresponding to the very dense barium plasma region $\alpha \gg 1$ is

$$
\omega+\mathbf{k} \cdot \mathbf{v}_{s w}=\frac{(1+i)}{\sqrt{2}} \sqrt{\frac{8}{\pi}} \alpha_{o}^{\frac{1}{2}} \omega_{p p} \frac{k v_{T p}}{\left|\mathbf{k} \cdot \mathbf{v}_{s w}\right|}
$$

which yields a growth rate $\gamma \propto n_{B}^{\frac{1}{2}}$ as in the previous case. Hence contrary to the lower-hybrid mode the excitation of the ion acoustic mode takes place throughout the whole cloud where the solar wind is present. The maximum growth rate corresponds to short wavelength ion-acoustic oscillations with $k d_{*} \simeq 1$. The frequency of oscillations as measured by the spacecraft given by $\omega+\mathbf{k} \cdot \mathbf{v}_{s w}$, is of the order of the barium ion plasma frequency $\omega_{p B}$ which is of the order of $\mathrm{kHz}$ close to the compressed region.

\section{B) Current driven lower-hybrid mode instability.}

As mentioned before the large gradients of magnetic fields in the turbulent zone of the cloud result in diamagnetic currents flowing as a consequence of Ampere's law. The effect of plasma and magnetic field inhomogeneities on the lower-hybrid drift instability have been considered by Davidson et $\mathrm{al}^{11}$ and Hsia et $\mathrm{al}^{13}$ The lower-hybrid drift instability initiated by the current which has been investigated Sotnikov et $\mathrm{al}^{10}$ is of a kinetic nature which has also been analysed by Davidson et $\mathrm{al}^{11}$. Due to the presence of cold barium ions there also appears a hydrodynamic instability of the lower-hybrid drift mode described by the following dispersion relation in the solar wind frame 


$$
\frac{\omega_{p e}^{2}}{\omega_{H e}^{2}}\left(1+\frac{\omega_{p e}^{2}}{k^{2} c^{2}}\right)-\frac{\omega_{p B}^{2}}{\left(\omega+\mathbf{k} \cdot \mathbf{v}_{s w}\right)^{2}}-\frac{\omega_{p e}^{2} k_{y} \kappa}{\omega_{H e}\left(\omega-k_{y} u_{e y}\right) k^{2}}+\frac{1}{k^{2} d_{p}^{2}}=0
$$

Here we have assumed that the mode under consideration is polarized in the plane perpendicular to the magnetic field with $k_{\|}=0$. The inhomogeneity of the density and the magnetic field profiles perpendicular to the magnetic field is characterised by the inhomogeneity scale length $\kappa^{-1}$ defined by

$$
\kappa=\frac{1}{n_{o}} \frac{d n_{o}}{d x}-\frac{1}{H} \frac{d H}{d x}
$$

and the electron current velocity $u_{e y}$ that supports the inhomogeneity of the magnetic field is

$$
u_{e y}=\frac{c}{4 \pi e n_{e}} \frac{d H}{d x}
$$

In the case of sufficiently small $\kappa$ the lower-hybrid drift instability is of a resonant nature where

$$
\omega-k_{y} u_{e y}=\varepsilon,|\varepsilon| \ll \omega
$$

In this case we have from Eq.(16) the following equation for the frequency of the unstable mode

$$
\omega=-\mathbf{k} \cdot \mathbf{v}_{s w}+\omega_{s p} \equiv-\mathbf{k} \cdot \mathbf{v}_{s w}+\omega_{L H B} k r^{*} \sqrt{\frac{n_{B}}{n_{e}}} / \sqrt{1+\beta+k^{2} r^{* 2}}
$$

and its growth rate $\gamma=\operatorname{Im} \varepsilon$ where

$$
\varepsilon^{2}=\frac{n_{p}}{n_{B}} \frac{m_{B}}{m_{p}} \frac{k_{y} \kappa}{k^{2}} \frac{\omega_{s p}^{3}}{\omega_{H p}}
$$

here $\omega_{s p}$ is the frequency measured by the spacecraft, and the following notation has been used

$$
r^{*}=\sqrt{\frac{T_{p}}{m_{e}}} \frac{1}{\omega_{H e}} \sqrt{\frac{n_{e}}{n_{p}}}, \beta=\frac{T_{p}}{m_{e} v_{A e}^{2}} \frac{n_{e}}{n_{p}}
$$

where $v_{A e}=c \omega_{H e} / \omega_{p e}$ is the electron Alfven velocity, $\omega_{L H B} \approx \sqrt{\frac{m_{p}}{m_{B}}} \omega_{L H}$ - is the lowerhybrid frequency for barium ions. The typical value of the frequency in the spacecraft reference frame in the limit $k r^{*}>1$ does not exceed $\frac{1}{2 \pi} \omega_{L H B}$ or $(10-15) \mathrm{Hz}$. 
The mechanism of the instability is quite a simple one. The necessary condition for the instability is $\kappa<0$ giving rise to a negative energy drift mode and any dissipation mechanism connected with the absorption of energy by barium ions or by solar wind protons leads to an instability. In the case of the hydrodynamical instability the absorption of energy by cold barium ions is important. In obtaining the growth rate Eq.18 we have neglected the input from the wave damping due to the solar wind protons, the condition for this is

$$
\frac{|\varepsilon|}{\omega} \gg \frac{n_{p}}{n_{B}} \frac{m_{B}}{m_{p}}\left(\frac{\omega_{s p}}{k v_{T p}}\right)^{3}
$$

which is more easily fulfilled in the region of dense barium plasma. The growth rate of the hydrodynamical lower-hybrid drift instability for $k r^{*}<1$ (increasing with $\left.n_{B}\right)$ is $\gamma \sim n_{B}^{\frac{1}{4}}$. For example let us consider the region of dense plasma and "piledup" magnetic field $n_{e} \simeq n_{B} \simeq 10^{4} \mathrm{~cm}^{-3}, H \simeq 1.5 \times 10^{2} n T$. In that region $\omega_{p e} / 2 \pi \simeq$ $\frac{1}{2} \times 10^{6} \mathrm{~Hz}, \omega_{\mathrm{He}} / 2 \pi \simeq 4 \times 10^{2} \mathrm{~Hz}$ the electron current velocity $u_{e y} \approx 10^{6} \mathrm{~cm} / \mathrm{s}$, we used here also $\Delta x \simeq 3 \mathrm{~km}$ for the characteristic space scale of the inhomogeneity in the "piled-up" region. The growth rate obtained from the Eq.(18) is $\gamma \simeq 10^{-1} s^{-1}$ and the typical growth length of the oscillations is $L \simeq \frac{10 v_{s w}}{\gamma} \sim 10^{7} \mathrm{~cm}$ which is quite sufficient for the development of the instability. The experimental results ${ }^{5,7}$ shows the presence of intense oscillations down to the frequency of the lower-hybrid barium resonance in the region containing the whole turbulent zone up to the boundary of the cavity.

In the case of the kinetic drift instability it is necessary to take into account the wave damping due to solar wind protons in the dispersion relation given by Eq.(16) and subsequently to make the following substitution

$$
\frac{1}{k^{2} d_{p}^{2}} \rightarrow \frac{1}{k^{2} d_{p}^{2}}\left(1+i \sqrt{\frac{\pi}{2}} \frac{\omega}{k v_{T p}}\right)
$$

Since the excited waves have negative energy the wave damping leads to the instability but of a kinetic nature with the growth rate similar to that obtained in ref. ${ }^{10}$ and given by

$$
\gamma=\operatorname{Im} \varepsilon=i \sqrt{\frac{2}{\pi} \frac{m_{B}}{m_{p}} \frac{n_{e}}{n_{B}}} \kappa r_{L p} \frac{\omega_{L H} k_{y} r^{*}}{\sqrt{1+\beta+k_{y}^{2} r^{* 2}}}, r_{L p}=\sqrt{\frac{T_{p}}{m_{p}}} \frac{1}{\omega_{H p}}
$$

In Conclusion we find 
1. The beam driven lower-hybrid instability is possible only in the outer part of barium cloud where $n_{B}<n_{p} m_{p} / m_{B}$

2. The main instability due to the barium beam, solar wind plasma interaction is the ion acoustic instability, this is responsible for the wave excitation in the wide frequency range from tens of $\mathrm{Hz}$ up to $\mathrm{kHz}$.

3. The current driven lower-hybrid hydrodynamic drift instability is responsible for the low frequency (of the order of tens of $\mathrm{Hz}$ or less) part of the spectrum in the whole of the turbulent zone.

V. Nonlinear theory of the instabilities involved, estimation of wave amplitudes, and energetic electron tails.

It has been shown in the previous section that the main wave activity in AMPTE is connected with two instabilities namely the current driven lower-hybrid drift instability for the low frequency range and the beam driven ion acoustic instability for the high frequency part of the spectrum. We present here estimates that give us the opportunity to compare the theoretical treatment with the observations. The detailed nonlinear theoretical analysis of the instabilities involved would be the subject of futher work and here we restrict ourselves with a brief nonlinear description. For the lower-hybrid drift instability we will consider the most important nonlinear process to be the modulational instability. Due to this instability waves that have been initially excited in the plane perpendicular to the magnetic field acquire a component of the wave vector along the magnetic field such that

$$
k_{\|} \sim k \sqrt{\frac{m_{e}}{m_{B}}} \sim \frac{\omega_{L H}}{v_{T e}}
$$

This results in the cascade of waves with high phase velocities along $\underline{B}$ to waves with phase velocities along $\mathbf{B}$ close to the electron thermal velocity and strong damping of the oscillations by electrons freezes the wave amplitude level at the level of the threshold of the modulational instability.

\section{A) Modulational instability of lower-hybrid waves.}

We will consider the case when the monochromatic lower-hybrid wave excited by the drift instability and polarized in the plane perpendicular to magnetic field acts as a pump for the parametric instability and generation of oblique $\left(k_{\|} \neq 0\right)$ waves. In that case the wave potential can be written in the form 


$$
\phi=e^{i\left(\mathbf{k}_{o} \cdot \mathbf{r}_{\perp}-\omega_{o} t\right)}\left[\phi_{o}+\phi_{+} e^{i\left(\mathbf{k}_{\perp} \cdot \mathbf{r}_{\perp}+k_{z} z-\omega t\right)}+\phi_{-} e^{-i\left(\mathbf{k}_{\perp} \cdot \mathbf{r}_{\perp}+k_{z} z-\omega t\right)}\right]
$$

Here $\mathbf{k}_{o}$ and $\omega_{o}$ are the wave vector and the frequency of the pump wave and

$$
\omega_{o}=-\mathbf{k}_{o} \cdot \mathbf{v}_{s w}+\omega_{L H B}
$$

which follows from Eq.(16) in the limit $\kappa r^{*}>1$ ie when the proton term is unimportant. Due to the modulational instability two satellites $\phi_{+}, \phi_{-}$with the wave vectors $\mathbf{k}_{o} \pm \mathbf{k}$ are excited resulting in the modulation of the pump wave amplitude. An analysis of the experimental data demonstrates that in the turbulent zone the magnetic field develops fine structure ${ }^{9}$. Such structure is due to the modulation of the magnetic field that has a characteristic space scale perpendicular to the magnetic field direction and very prolonged along the field lines and varying on a time scale much faster than the barium gyroperiod. We assume that this modulation is created by long wavelength magnetosonic waves that parametrically couple the pump wave to the lower-hybrid satellites $\phi_{ \pm}$. Hence contrary to the usual treatment of the modulational instability of lower-hybrid waves in which the slow mode is connected with density perturbations such as the ion-acoustic mode we will consider the case when the slowly varying mode is associated with the magnetosonic mode and results in magnetic field modulations. In accordance with experimental data we will also assume that for these perturbations $k_{z} \ll k_{\perp}$. Using the drift approximation for electrons $\left|\frac{d}{d t}\right| \ll \omega_{H e}$ and supposing that ions are unmagnetized for the lower-hybrid mode $\left|\frac{d}{d t}\right| \gg \omega_{H B}$, it is possible to obtain the following equation for the electric field of lower-hybrid waves in the case of quasineutrality $n_{e}^{\prime}=n_{i}^{\prime}$ :

$$
\begin{gathered}
\left(\frac{\partial}{\partial t}+u_{e y} \frac{\partial}{\partial y}\right)^{2}\left[1+\frac{1}{\omega_{L H B}^{2}}\left(\frac{\partial}{\partial t}-\mathbf{v}_{s w} \cdot \underline{\nabla}\right)^{2}\right] \underline{\nabla} \cdot \mathbf{E}_{\perp}^{\prime}+\frac{m_{B}}{m_{e}}\left(\frac{\partial}{\partial t}-\mathbf{v}_{s w} \cdot \underline{\nabla}\right)^{2} \frac{\partial E_{z}^{\prime}}{\partial z}-\left(\frac{\partial}{\partial t}+u_{e y} \frac{\partial}{\partial y}\right) \\
\times\left(\frac{\partial}{\partial t}-\mathbf{v}_{s w} \cdot \underline{\nabla}\right)^{2} \kappa \frac{E_{y}^{\prime}}{\omega_{H B}}-\frac{1}{\omega_{H e}}\left(\frac{\partial}{\partial t}+u_{e y} \frac{\partial}{\partial y}\right)\left(E_{x}^{\prime} \frac{\partial}{\partial y}-E_{y}^{\prime} \frac{\partial}{\partial x}\right)\left(\frac{\delta H}{H_{o}}-\frac{\delta n}{n_{o}}\right)=0 \quad(20)
\end{gathered}
$$

Here the main nonlinear term is the $\left(\mathbf{v}_{e} \cdot \underline{\nabla}\right) \mathbf{v}_{e}$ nonlinearity in the electron equations of motion, $\delta n$ and $\delta H$ are the density and magnetic field modulation due to the slow magnetosonic wave. Neglecting in the Eq.(20) the term proportional to $\kappa$ that is responsible for the drift instability of the pump wave it is possible to obtain the following 
relation for the wave potential of the two lower-hybrid satellites

$$
\begin{gathered}
\phi_{+}=-\frac{i \omega_{L H B}^{2}\left[\mathbf{k}_{o} \times \mathbf{k}\right]_{z}}{\omega_{H B}\left(\omega-k_{y} u_{e y}\right)} \frac{\phi_{o}}{\left(\mathbf{k}_{o}+\mathbf{k}\right)^{2}}\left(\frac{\delta H}{H_{o}}-\frac{\delta n}{n_{o}}\right) \times \frac{1}{\delta_{+}-2 \frac{\left(\omega+\mathbf{k} \cdot \mathbf{v}_{s w}\right)}{\omega_{L H B}}} \\
\phi_{-}=-\frac{i \omega_{L H B}^{2}\left[\mathbf{k}_{o} \times \mathbf{k}\right]_{z}}{\omega_{H B}\left(\omega-k_{y} u_{e y}\right)} \frac{\phi_{o}}{\left(\mathbf{k}_{o}-\mathbf{k}\right)^{2}}\left(\frac{\delta H^{*}}{H_{o}}-\frac{\delta n^{*}}{n_{o}}\right) \times \frac{1}{\delta_{-}+2 \frac{\left(\omega+\mathbf{k} \cdot \mathbf{v}_{s w}\right)}{\omega_{L H B}}}
\end{gathered}
$$

where we have used the fact that for the pump wave resonance $\omega_{o}=k_{y o} u_{e y}$ and

$$
\delta \pm=\frac{m_{B}}{m_{e}} \frac{k_{\|}^{2}}{\left(\mathbf{k}_{o} \pm \mathbf{k}\right)^{2}} \frac{\omega_{L H B}^{2}}{\left(\omega-k_{y} u_{e y}\right)^{2}}
$$

is the detuning between the frequency of the pump wave and the satellites. For simplicity we will suppose that the lower-frequency mode is mainly inhomogeneous along the direction of the current $\left(k_{y} \neq 0\right)$ and very prolonged along the magnetic field $\left(k_{\|} \ll k_{y}\right)$. The equation connecting the density and magnetic field fluctuations is the usual one for magnetosonic waves:

$$
\left(\frac{\partial}{\partial t}-\mathbf{v}_{s w} \cdot \underline{\nabla}\right)^{2} \delta n=v_{A B}^{2} \frac{n_{o}}{H_{o}} \frac{\partial^{2} \delta H}{\partial y^{2}}
$$

$v_{A B}=\sqrt{\frac{H}{4 \pi n_{B} m_{B}}}$ is the Alfven velocity for barium ions.

While obtaining the equation for $\delta H$ it is necessary to use the following relation between the electron and ion velocities in the direction of inhomogeneity

$$
\left(\frac{\partial}{\partial t}+u_{e y} \frac{\partial}{\partial y}\right) \delta v_{y i}=\left(\frac{\partial}{\partial t}-\mathbf{v}_{s w} \cdot \underline{\nabla}\right) \delta v_{y e}
$$

This relation being the consequence of quasineutrality of the slow magnetosonic wave ie. $\delta n_{e}=\delta n_{i}$. Then the equation for $\delta H$ has the following form:

$$
\begin{aligned}
& \left(\frac{\partial}{\partial t}+u_{e y} \frac{\partial}{\partial y}\right)\left[\frac{\partial^{2} \delta H}{\partial y^{2}}-\frac{1}{v_{A B}^{2}}\left(\frac{\partial}{\partial t}-\mathbf{v}_{s w} \cdot \underline{\nabla}\right)^{2} \delta H\right]= \\
& -\frac{4 \pi e n_{o} c}{H_{o}^{2} \omega_{L H B}^{2}}\left(\frac{\partial}{\partial t}-\mathbf{v}_{s w} \cdot \underline{\nabla}\right)^{2}\left(\frac{\partial \varphi^{\prime}}{\partial x} \frac{\partial}{\partial y}-\frac{\partial \varphi^{\prime}}{\partial y} \frac{\partial}{\partial x}\right) \frac{\partial^{2} \varphi^{\prime}}{\partial y^{2}}
\end{aligned}
$$


The relation for the magnetic field modulation resulting from the coupling between the pump lower hybrid wave and the satellites follows from Eq. 22 and is given by

$$
\begin{aligned}
\delta H= & \frac{4 \pi n_{o} c}{H_{o}^{2}} \frac{i}{\omega_{L H B}^{2}}\left[\mathbf{k}_{o} \times \mathbf{k}\right]_{z} \frac{\left(\omega+\mathbf{k} \cdot \mathbf{v}_{s w}\right)^{2}}{\left(\omega-k_{y} u_{e y}\right)} \times \frac{1}{1-\frac{\left(\omega+\mathbf{k} \cdot \mathbf{v}_{s w}\right)^{2}}{k_{y}^{2} v_{A B}^{2}}} \\
& \times\left[\left(\varphi_{+} \varphi_{o}^{*}-\varphi_{-}^{*} \varphi_{o}\right)+\frac{2 k_{o y}}{k_{y}}\left(\varphi_{+} \varphi_{o}^{*}+\varphi_{-}^{*} \varphi_{o}\right)\right]
\end{aligned}
$$

We restrict ourselves to the consideration of the case when as the result of the modulation sufficiently long wavelength magnetic structures are created $k_{y} \ll k_{o y}$. In that case the dispersion relation has a following form

$$
1=8 \frac{\omega+\mathbf{k} \cdot \mathbf{v}_{s w}}{\omega_{L H B}} \frac{k_{o y}}{k_{y}}\left[\mathbf{k}_{o} \times \mathbf{k}\right]_{z}^{2} \frac{c^{2}\left|\varphi_{o}\right|^{2}}{H_{o}^{2}} \times \frac{1}{\left(\omega-k_{y} u_{e y}\right)^{2}} \cdot \frac{1}{\delta_{o}^{2}-4\left(\omega-k_{y} u_{e y}\right)^{2} / \omega_{L H B}^{2}}
$$

It follows from the dispersion relation that the maximum growth rate corresponds to the resonant case $k_{y} u_{e y}+\mathbf{k} \cdot \mathbf{v}_{s w}=0$.

For sufficiently strong pump when the condition

$$
\frac{k_{o y}}{k_{y}} \frac{c^{2} E_{o}^{2}}{H_{o}^{2}}>\frac{\omega_{L H B}^{2}}{k^{2}} \frac{m_{B}}{m_{e}} \frac{k_{\|}^{2}}{k^{2}}
$$

is fulfiled the maximum growth rate obtained from Eq. (24) is equal to:

$$
\gamma_{\max }=\frac{\sqrt{3}}{2^{2 / 3}}\left[\omega_{L H B} \frac{k_{o y}}{k_{y}}\left[\mathbf{k}_{o} \times \mathbf{k}\right]_{z}^{2} \frac{c^{2}\left|\varphi_{o}\right|^{2}}{H_{o}^{2}}\right]^{1 / 3}
$$

The magnetic structures arising as the result of modulational instability are convected with the solar wind and in order to give the instability sufficient time to grow the following condition must be fullfiled $\gamma_{\max } L / v_{s w}>10$, where $L$ is the size of the cloud.

For $L \sim 100 \mathrm{~km}, v_{s w} \sim 3.10^{7} \mathrm{~cm} / \mathrm{sec}$ we need a value of $\gamma_{\max } \sim 30 \mathrm{H}_{z}$ and it follows from the Eq. (26) that for $H=3.10^{-3} G u_{e y} \sim 10^{6} \mathrm{~cm} / \mathrm{s}, k_{o} \simeq \frac{\omega_{L H B}}{u_{e}} \sim 10^{-4} \mathrm{~cm}^{-1}$ and $k / k_{o} \sim \frac{1}{5}$ the typical lower-hybrid wave amplitude needed for the development of the instability is $E_{o} \sim(3-5) \mathrm{mV} / \mathrm{m}$ which is close to the observed values. The modulational 
instability not only results in the creation of magnetic structures slowly varying with time but also in the appearance in the lower-hybrid wave spectrum waves with $k_{\|} \neq 0$. The waves with $k_{\|} \sim \frac{1}{3 v_{T e}} \omega_{L H B}$ or $\frac{k_{\|}}{k_{o}} \sim \frac{u_{e y}}{3 v_{T e}} \sim \frac{1}{3} \cdot 10^{-2}$ will be efficiently absorbed by resonant electrons, this absorption will prevent growth of the pump amplitude causing saturation of the pump wave.

\section{(B) Electron heating.}

Since the parametrically excited lower-hybrid waves have a finite longitudinal electric field $E_{\|}$, they can stochastically accelerate electrons that fall into Cherenkov resonance $\left(\omega=k_{\|} v_{\|}\right)$with them. The detailed calculation of this effect will be presented in a future paper. Here will give an estimate for the energy gained by these resonant electrons based on the quasi-linear diffusion equation

$$
v_{e} \frac{\partial f}{\partial z}=\frac{\partial}{\partial v_{\|}} D_{\|} \frac{\partial f_{e}}{\partial v_{\|}}
$$

where $f_{e}$ is the electron distribution function and $D_{\|}$is the diffusion coefficient in the parallel direction given by

$$
D_{\|}=\frac{e^{2}}{m_{e}^{2}}\left|E_{k}\right|^{2}\left(\frac{k_{\|}}{k}\right)^{2} \frac{1}{v_{\|}}, \quad\left(k_{\|}=\omega_{L H B} / v_{\|}\right)
$$

It is easy to obtain the following scaling law for the characteristic energy gained by resonant electrons while flowing through the turbulent zone

$$
\mathcal{E}_{e} \simeq\left(\frac{\omega_{p e}^{2}}{\omega_{H e}^{2}} \frac{|E|^{2}}{4 \pi n_{e}} m_{e} v_{A e}^{2} \omega_{L H B} L \sqrt{m_{e}}\right)^{\frac{2}{5}}
$$

Using the following values, for the magnetic field, plasma electron density and turbulent wave field of $H \sim 100 n T, n_{e} \simeq 10^{2}$ and $E \sim 10 \mathrm{mV} / \mathrm{m}$ and distance $L$ representing the size of the turbulent zone of order $300 \mathrm{~km}$ we have from equation (28) an estimate of the typical energies of the accelerated electrons to be $\sim 100 \mathrm{eV}$ which agrees very well with the observations ${ }^{6}$. We can also estimate the fraction of electrons accelerated in the tail by equating the rate at which energy is cascading into larger parallel wavenumbers $k_{\|}$as a result of the modulational instability to the Landau damping rate of these waves by electrons. The Landau damping rate of waves on electrons is given by

$$
\gamma_{e} \simeq \omega_{L H} \frac{n_{T A I L}}{n_{o}} \frac{\omega_{L H}^{2}}{k_{\|}^{2} v_{T e}^{2}}
$$


This results in $\frac{n_{\text {TALL }}}{n_{o}}$ of order $\frac{\gamma_{\max }}{\omega_{L H}}$ where $\gamma_{\max }$ is modulational growth rate defined by eq. (26), resulting in a $n_{T A I L} / n_{o}$ of about $1-10 \%$.

\section{(C) Ion Acoustic Waves}

The instability which drives the ion-acoustic waves unstable is of the dissipative type and is caused by the cold beam of barium ions flowing through the solar wind plasma. Here we present a qualitative description of the dynamics for the case $\alpha_{o}<1$. The energy source of the instability is the relative flow between the solar wind and the released ions. For the situation we consider ie when $n_{p} m_{p} \ll n_{B} m_{B}$ no significant change occurs in the barium ion velocity distribution. The velocity spread of the barium ions caused by the instability cannot exceed the value $\Delta v / v \leq \frac{n_{p}}{n_{B}} \frac{m_{p}}{m_{B}}<\left(10^{-3}-10^{-4}\right)$. In this case the main quasilinear effect that can stabilize the wave growth is the formation of a plateau on the resonant proton distribution function. The formation of a plateau switches off the dissipation mechanism. Since for oblique ion-acoustic waves the whole bulk of thermal protons is in resonance with the waves and the energy gained by these particles due to plateau formation is

$$
W_{p} \simeq n_{o} T_{p}
$$

It follows from the condition of instability ie., Eq.(14) that the excited waves are propagating almost perpendicular to the direction of relative motion $\theta=\frac{\mathbf{k} \cdot \mathbf{v}_{s w}}{\left|\mathbf{k} \mathbf{v}_{s w}\right|} \simeq$ $\alpha_{o}^{\frac{1}{2}} \frac{\omega_{p p} d_{*}}{v_{s w}} \simeq \sqrt{\frac{T_{e}}{m_{B} v_{s w}^{2}}} \ll 1$. This results in predominantly elastic scattering of the barium ion beam by waves. The change of longitudinal energy of the beam in such an elastic scattering process is equal to

$$
\Delta W_{B} \simeq n_{B} m_{B} v_{s w} \Delta v_{B} \simeq \eta n_{p} m_{p} v_{s w}^{2}
$$

which also corresponds to momentum balance between the barium ions and the solar wind, where $\eta$ is the fraction of solar wind momentum lost in this interaction.

Only small amounts of this energy $\Delta W_{B}$ of order of $\theta$ is going into the waves, hence

$$
W_{E} \simeq \sqrt{\frac{T_{e}}{m_{B} v_{s w}^{2}}} \Delta W_{B}
$$

is the energy in the waves.

Since $\omega / k v_{T p} \simeq \alpha_{o}^{\frac{1}{2}} \omega_{p p} \frac{d_{*}}{V_{T_{p}}} \simeq \sqrt{\frac{T_{e}}{T_{p}} \frac{m_{p}}{m_{B}}} \ll 1$ the interaction of these waves with the thermal protons is also almost an elastic one. The energy in the waves $W_{E}$ is small in 
comparison with the energy gained by thermal protons in the process of their quasilinear diffusion just by the factor $\omega / k \mathbf{v}_{T_{p}}$ ie.

$$
W_{p} \simeq W_{E} \sqrt{\frac{T_{p}}{T_{e}} \frac{m_{B}}{m_{p}}}
$$

Using the value of $W_{p}$ from the Eq. (30) it is easy to obtain the final result for thermal proton heating:

$$
T_{p} \simeq \eta^{2} m_{p} v_{s w}^{2}
$$

If we assume that the $70 \%$ of the solar wind energy lost is due to the instability under consideration then $\eta \simeq 2 / 3$ and it follows from Eq. (34) that $T_{p} \simeq 0.65 k \mathrm{eV}$ which is somewhat larger than the observed value of $T_{p} \simeq 0.4 \mathrm{keV}$. The latter corresponds to $\eta \simeq 0.5$ and may serve as an indication that the decceleration of the solar wind flow in the compressed region by the DC electric field, due to gradients in the magnetic field is also important. The wave energy obtained from the Eqs. (33), (34) given by

$$
W_{E}=\frac{E^{2}}{4 \pi} \simeq \eta^{2} \sqrt{\frac{m_{p} T_{e}}{m_{B} T_{p}}} n_{p} m_{p} v_{s w}^{2}
$$

is small in comparison with the energy lost by the solar wind flow by the factor $\sqrt{m_{p} / m_{B}}$. Nevertheless the wave electric field calculated from the Eq. (35) for the typical solar wind parameters $n_{p} \approx 5 \mathrm{~cm}^{-3}, \mathbf{v}_{s w} \simeq 270 \mathrm{~km} / \mathrm{s}$ and $\eta \simeq 0.5$ is quite large and corresponds to spectral energy density in the $k H z$ range $\sqrt{E_{f}^{2}}$ of about $30 \mathrm{mV} / \mathrm{m} \sqrt{\mathrm{HZ}}$ which agrees with the observed values.

\section{Conclusions}

In this paper we have proposed possible mechanisms for the generation of the broadband wave turbulence observed in the AMPTE release experiments. The mechanisms are based on two types of instabilities that are possible in the cloud of the expanding barium ions interacting with the solar wind. One is the barium beam driven instability resulting in the excitation of long wavelength $(\lambda \geq 1 \mathrm{~km})$ whistler type waves as well as short wavelength $(\lambda \leq 10-100 \mathrm{~m})$ ion-acoustic oscillations. Another is the lower-hybrid drift instability driven by currents associated with the large magnetic field gradients in the turbulent zone. The frequencies of the excited waves change from about 5-10 $\mathrm{Hz}$ in the lower-hybrid range up to $1 \mathrm{kHz}$ for ion acoustic waves. The analysis of the instabilities presented shows that 
1. The modulational instability of lower-hybrid waves could result in magnetic field modulation aligned along the magnetic field direction, of fine magnetic structures which are observed both in the experiment ${ }^{9}$ and in the simulations ${ }^{14}$

2. Excitation due to the modulational instability of oblique lower-hybrid waves with $k_{\|} \neq 0$ producing a cascade in wavenumber to larger values leads to the absorption of the wave energy by resonant electrons producing energetic field aligned electron tails with energies greater than $100 \mathrm{eV}$.

3. The nonlinear estimates derived from the saturation of the wave spectrum by the modulational instability for lower-hybrid waves of the electric field amplitude give values of $E$ in the range $5-15 \mathrm{mV} / \mathrm{m}$. For the ion acoustic waves which heat the ions the estimated electric field amplitude is $E \sim 300 \mathrm{mV} / \mathrm{m}$ which is in very good agreement with the experiment.

4. The wave energy is not enough for significant "pick-up" of the barium ions by the solar wind some of the heating of the barium ions may be mainly due to the lowerhybrid drift instability. This is the dissipative instability of the negative energy waves resonant with the thermal ions, with the growth of the wave amplitude resulting in energy gain of the thermal particles. The same physics applies in respect to the ion-acoustic instability which is also a negative energy instability and could serve as the reason for the significant heating of the thermal solar wind protons. 


\section{CAPTIONS}

Figure 1. Graphical representation of the dispersion relation $F(\omega)$, showing $F_{\min }$ values of $F(\omega)$ falling below this value indicate instability.

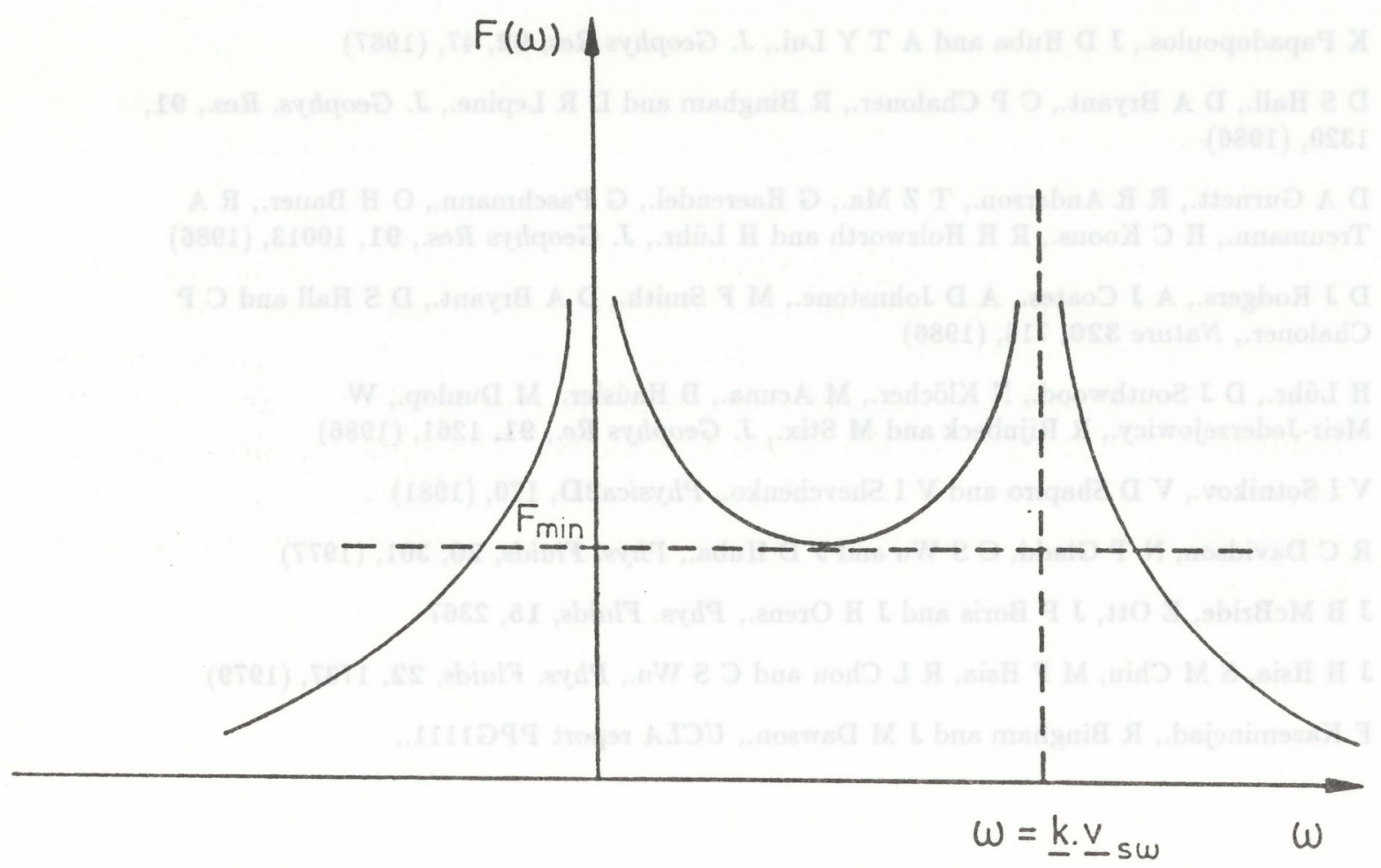




\section{REFERENCES}

1. W I Ip and W I Axford., Comets, L L Wilkering, (University of Arizona, Tuscon), 588, (1982)

2. T E Hartle., K W Ogilvie and C S Wu., Planet Space Science, 21, 2181, (1973)

3. C S Wu., R E Hartle and K Ogilvie., J. Geophys Res. 78, 306, (1973)

4. Bingham R., Bryant D A., Hall D S., J M Dawson., F Kazeminejad and J J Su., Computer Phys. Com. 49, 257 (1988)

5. K Papadopoulos., J D Huba and A T Y Lui., J. Geophys Res, 92, 47, (1987)

6. D S Hall., D A Bryant., C P Chaloner., R Bingham and D R Lepine., J. Geophys. Res., 91, $1320,(1986)$

7. D A Gurnett., R R Anderson., T Z Ma., G Haerendel., G Paschmann., O H Bauer., R A Treumann., H C Koons., R H Holzworth and H Lühr., J. Geophys Res., 91, 10013, (1986)

8. D J Rodgers., A J Coates., A D Johnstone., M F Smith., D A Bryant., D S Hall and C P Chaloner., Nature 320, 716, (1986)

9. H Lühr., D J Southwood., N Klöcher., M Acuna., B Haúsler., M Dunlop., W Meir-Jederzejowicy., R Rijnbeck and M Stix., J. Geophys Re., 91, 1261, (1986)

10. V I Sotnikov., V D Shapiro and V I Shevchenko., Physica2D, 170, (1981)

11. R C Davidson, N T Gladd, C S Wu and J D Huba., Phys. Fluids, 20, 301, (1977)

12. J B McBride, E Ott, J P Boris and J H Orens., Phys. Fluids, 15, 2367

13. J H Hsia, S M Chiu, M F Hsia, R L Chou and C S Wu., Phys. Fluids, 22, 1737, (1979)

14. F Kazeminejad., R Bingham and J M Dawson., UCLA report PPG1111.. 


